$\underline{\xi}=$

\title{
Ethylene glycol poisoning treated using combination of ethanol and haemodialysis therapy instead of fomepizole: a case report
}

\author{
Kazunobu Une ${ }^{1}$, Yasumasa Kishi $^{2}$, Yui Hidaka ${ }^{3}$, Takashi Esaki ${ }^{3}$ \\ ${ }^{1}$ Department of Emergency Medicine, JA Onomichi General Hospital, 1-10-23 Hirahara, Onomichi, Hiroshima 722-8508, Japan \\ 2 JA Onomichi General Hospital, 1-10-23 Hirahara, Onomichi, Hiroshima 722-8508, Japan \\ ${ }^{3}$ Division of Critical Care Medicine, University of Occupational and Environmental Health, 1-1 Iseigaoka, Yahatanishi-ku, Kitakyushu, \\ Fukuoka 807-8555, Japan \\ ${ }^{4}$ Department of Nephrology, JA Onomichi General Hospital, 1-10-23 Hirahara, Onomichi, Hiroshima 722-8508, Japan \\ *Corresponding author E-mail: ichinonki212@yahoo.co.jp
}

\begin{abstract}
We report a case of ethylene glycol poisoning in a 70-year-old man with headache and dysarthria. Blood tests showed a marked increase in the anion gap $(29.8 \mathrm{mOsm} / \mathrm{kg})$ and osmotic gap $(14 \mathrm{mOsm} / \mathrm{kg})$. The estimated blood concentration of ethylene glycol was $86.8 \mathrm{mg} / \mathrm{dL}$. Ethanol was administered and he underwent haemodialysis for 7 cycles, which was discontinued on day 12. Fomepizole, which competes with alcohol dehydrogenase, is an effective treatment for ethylene glycol poisoning. However, it is usually prescribed within $24 \mathrm{~h}$ after poisoning, and studies on its use in cases of severe acute renal injury over time are limited. We were able to obtain good results with haemodialysis without using fomepizole. Conventional treatments such as haemodialysis may be more useful than fomepizole in terms of cost benefits in patients with addiction who have been taking the drug for a long time or who have advanced renal injury.
\end{abstract}

Keywords: Acute Kidney Disease; Cost Benefit; Ethylene Glycol Poisoning; Fomepizole; Haemodialysis.

\section{Introduction}

Ethylene glycol poisoning is mainly caused by the ingestion of antifreeze during suicide attempts or accidents. The symptoms include rapidly progressing metabolic acidosis, central nervous system dysfunction, and acute kidney disease due to acute tubular necrosis. Ethylene glycol poisoning is a relatively rare form of poisoning; the absence of specific symptoms often makes it difficult to diagnose it immediately, and delays in diagnosis frequently lead to fatal outcomes. Treatment includes using ethanol as an antidote and performing haemodialysis to alleviate acidosis and acute kidney injury. However, since 2015, fomepizole, an alcohol dehydrogenase inhibitor covered by insurance, has been employed. The standard treatment procedure has also changed significantly (Kiyota 2016). There are many reports showing that fomepizole is effective in treating ethylene glycol poisoning (Amano et al. 2018, Nosaka and Satou 2019). However, in many clinical situations, this drug is not readily available in hospitals because it is very expensive and not used frequently. Here, we report a case of acute kidney disease caused by ingestion of ethylene glycol, which was treated by administration of ethanol and haemodialysis without using fomepizole. Although some reports show that fomepizole improves the prognosis for severe acute kidney disease, conventional treatment using ethanol may help save lives. Further, families of patients involved in suicide cases may have financial issues; therefore, it is important to consider the indications and cost-effectiveness of expensive drugs before administering them, as depicted in this case.

\section{Case presentation}

A 70-year-old man presented with decreased consciousness, headache, and dysarthria in late February 20XX. He had a medical history of depression, alcoholism, and chronic hepatitis. Although he showed no symptoms of limb paralysis, we performed computed tomography and magnetic resonance imaging of the brain because we suspected cerebral infarction. However, there were no obvious abnormal findings. He also had mild acidosis and renal damage, with a creatinine level of approximately $1.5 \mathrm{mg} / \mathrm{dL}$. Since we could not arrive at a definitive diagnosis, the patient was hospitalised for follow-up. The next day, a blood test revealed rapid progression of acidosis, renal dysfunction, and oliguria. Therefore, the patient was referred to our critical care centre where his height and weight were registered as 67 $\mathrm{cm}$ and $63 \mathrm{~kg}$, respectively. His consciousness level was 8 on the Glasgow Coma Scale (Eye, 2; Verbal, 1; Mouth, 5), blood pressure was $186 / 110 \mathrm{mmHg}$, pulse was $116 \mathrm{bpm}$ (sinus rhythm), axillary temperature was $36.1^{\circ} \mathrm{C}$, respiratory rate was $14 \mathrm{breaths} / \mathrm{min}$, and oxygen saturation was $100 \%\left(5 \mathrm{~L} \mathrm{O}_{2} / \mathrm{min}\right)$, with an aromatic odour accompanying heavy breathing. Neurological examinations revealed bilateral 
slow reflexes without stiff neck while the pupil diameters $(2.0 \mathrm{~mm} / 2.0 \mathrm{~mm})$ showed no laterality. Blood and urine test results, shown in Table 1, indicated unknown crystals in the urine (3+), marked rise of 29.8 in the anion gap (AG), and osmotic gap of $14 \mathrm{mOsm} / \mathrm{kg}$.

Table 1: Blood test results at admission



WBC, white blood cells; RBC, red blood cells; Hb, hemoglobin; Hct, hematocrit; PLT, platelets; PT, prothrombin; aPTT, activated partial thromboplastin time; Fib, fibrinogen; FDP, fibrin degradation products; HPF, high power field; TP, total protein; Alb, albumin; T-Bil, total bilirubin; AST, aspartate aminotransferase; ALT, alanine aminotransferase; LDH, lactate dehydrogenase; CK, creatine kinase; BUN, blood urea nitrogen; Cr; UA; CRP; C-reactive protein; $\mathrm{pO}_{2}$, partial pressure of oxygen; $\mathrm{pCO}_{2}$, partial pressure of carbon dioxide.

Considering the possibility of some kind of poisoning based on the medical history and blood tests, we conducted a medical interview with the family again and requested permission to search the patient's home. Meanwhile, blood tests and urinalysis were repeated at our hospital, and a nasogastric tube was inserted. Subsequently, approximately $30 \mathrm{~mL}$ of bile-like content with an aromatic odour was drained. In addition, urinalysis after hospitalisation revealed the presence of unknown crystals, and a blood test showed progression of acidosis with increased AG and renal dysfunction. Further, an empty container of antifreeze and suicide notes were found in the storeroom of the patient's home. We strongly suspected the possibility of ethylene glycol poisoning and estimated that approximately $40 \mathrm{~h}$ had passed since the oral ingestion. The concentration of ethylene glycol in blood was calculated as $86.8 \mathrm{mg} / \mathrm{dL}$. Renal dysfunction and anuria were observed, and there was no improvement in the consciousness level. Since fomepizole was not available, $50 \mathrm{~mL}$ of $40 \%$ ethanol was administered through the nasogastric tube as an antagonist to treat the poisoning. Additionally, haemodialysis was initiated to manage the severe renal damage. After $2 \mathrm{~h}$, the patient's consciousness level improved and he was able to communicate that he had attempted suicide. Although he refused treatment, he provided consent for temporary care and underwent dialysis for $5 \mathrm{~h}$ on the first day of hospitalisation. Although blood test results indicated a slight decrease in lactate level and an improvement in acidosis, the anuria persisted. The consciousness level did not decline again; however, the lactate level remained high and acidosis persisted. Despite an improvement in AG $(18 \mathrm{mOsm} / \mathrm{kg})$, there was still mild dilatation and an osmotic pressure gap of $6.5 \mathrm{mOsm} / \mathrm{kg}$, and treatment was continued. We again considered administration of fomepizole; nevertheless, it was not readily available in the hospital. Further, the patient and his family did not provide consent for the administration of this expensive drug because of financial constraints. We continued with the haemodialysis treatment after obtaining consent to maintain life support.

After the third day, the acidosis did not worsen, although renal dysfunction and oliguria persisted, and haemodialysis was continued on alternate days. After seven haemodialysis cycles, renal function and urine volume improved 12 days after admission (Figure 1), and haemodialysis was withdrawn. However, the patient was in an unstable state without clearly denying his attempt at suicide and was transferred to the hospital with his consent on day 15 for psychiatric treatment.

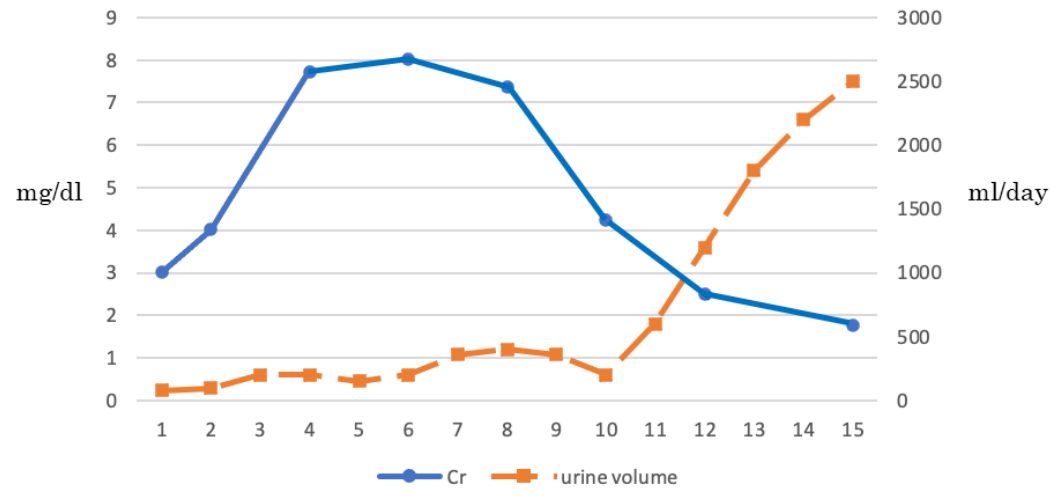

Fig. 1: Changes in Creatinine (Cr) and Urine Volume after Hospitalization. 


\section{Discussion}

The main concern with ethylene glycol poisoning is that its metabolites, glycolic acid and oxalic acid, cause metabolic acidosis, and the deposition of calcium oxalate in renal tubules causes renal damage (Howard et al. 1991). There have been many reports of cases of declined consciousness due to the suppression of the central nervous system as metabolic acidosis progresses and thereby requiring emergency care. In this case, ethylene glycol poisoning was diagnosed based on blood and urine examinations that revealed increased osmotic pressure gap measured as a result of the AG that caused metabolic acidosis and by interviewing the family after the patient's hospitalisation. In clinical practice, ethylene glycol poisoning has been reported in patients showing atypical signs such as facial nerve paralysis (Tanasescu et al. 2014), and it is likely that there are many situations in which diagnosis is difficult. While it is not easy to diagnose, ethylene glycol poisoning is associated with mortality rates between 1\% and 22\% (Basnayake et al. 2019). Therefore, it has been reported that early diagnosis and treatment are important factors because survival depends on the amount of ethylene glycol ingested and the time of initiation of treatment. In particular, the mortality rate is higher in cases where $\mathrm{pH}$ values are $<7.1$ and with more than $10 \mathrm{~h}$ of exposure (Basnayake et al. 2019). As with other cases of poisoning, it is important to accurately assess the situation and collect detailed information from the patient's family to make a prompt and appropriate diagnosis. Subsequently, blood and urine examinations should be performed to identify the causative substance and provide treatment. In cases of ethylene glycol poisoning, increased AG that causes metabolic acidosis, increased osmotic gap, characteristic drug crystals in the urine, and calcium oxalate crystals can be important diagnostic indicators (Yamada and Nishio 2004, Uchida et al. 2011). The osmotic pressure gap is the difference between the measured plasma osmotic pressure and the calculated osmotic pressure. It is used to estimate the concentration of ethylene glycol in the blood using its molecular weight in order to perform therapeutic intervention and provide a prognosis. Each formula is shown below (1):

- Osmotic pressure gap $=$ measured osmotic pressure $(\mathrm{Osm} / \mathrm{L})-$ calculated osmotic pressure $(\mathrm{Osm} / \mathrm{L})$.

- Osmotic pressure $(\mathrm{Osm} / \mathrm{L})=2 \times$ blood sodium concentration $(\mathrm{mEq} / \mathrm{L})+$ blood glucose level $(\mathrm{mg} / \mathrm{dL}) / 18+$ blood urea nitrogen value $(\mathrm{mg} / \mathrm{dL}) / 2.8$.

- Estimated concentration of ethylene glycol in the blood $(\mathrm{mg} / \mathrm{dL})=$ osmotic pressure gap $\times 62$ (molecular weight)/10.

Using the above formulae, the estimated blood concentration of ethylene glycol in this case was calculated as $86.8 \mathrm{mg} / \mathrm{dL}$. According to previous reports, ethylene glycol concentrations of $30-430 \mathrm{mg} / \mathrm{dL}$ in the blood and ingested doses in the range of $1.4-1.5 \mathrm{mg} / \mathrm{kg}$ are lethal (Uchida et al. 2011). Therefore, the present case could have been fatal if treatment had been delayed.

The purpose of the treatment is to slow down a series of toxic reactions by inhibiting the action of alcohol dehydrogenase, which is involved in the metabolism of ethylene glycol to glycolaldehyde. In Japan, fomepizole, an alcohol dehydrogenase inhibitor that has been covered by insurance since 2015, is used as the standard treatment for this type of poisoning. Before the advent of fomepizole, ethanol was used as an antagonist for this metabolic pathway. Ethanol has the advantage of being inexpensive and easily available. However, in addition to side effects such as nausea and vomiting, its efficacy is greatly affected by the patient's constitution, original drinking habits, and combined use of haemodialysis therapy. Moreover, the concentration of ethylene glycol in the blood needs to be frequently measured. There is no doubt that fomepizole plays a major role in the treatment of ethylene glycol poisoning in Japan. As a guideline, fomepizole is initiated in patients with blood ethylene glycol concentration $\geq 20 \mathrm{mg} / \mathrm{dL}$, metabolic acidosis, increased osmotic pressure gap, and visual field abnormality. Further, the actual event of poisoning should have been positively established and considered (Kiyota 2016). Nevertheless, fomepizole is a drug that is not readily available in hospitals because it is expensive, and further, ethylene glycol poisoning is rare (Ono et al. 2017). Hovda and Jacobsen (2008) have pointed out that although using this drug can reduce the need to initiate haemodialysis therapy, the duration and consequently the cost of hospitalisation can increase. In addition, there are many reports recommending that haemodialysis therapy should be started when blood concentration of ethylene glycol exceeds $50 \mathrm{mg} / \mathrm{dL}$ even if the patient is asymptomatic (Mégarbane 2010, Rietjens et al. 2014). A consensus statement published by Roberts et al. in 2015 also recommends intermittent haemodialysis for $8 \mathrm{~h}$ as empiric therapy when ethylene glycol levels in the blood cannot be measured. We decided to treat our patient with ethanol and haemodialysis rather than fomepizole because the latter was not immediately available in the hospital and also considering the financial circumstances of the patient and his family. Medically, haemodialysis was considered unavoidable in this case because it was estimated that $40 \mathrm{~h}$ had passed since ethylene glycol intake, based on its concentration in the blood. In many studies that reported the efficacy of fomepizole, the time period between ethylene glycol ingestion and treatment was as short as $24 \mathrm{~h}$ or less, while in our case, at least $40 \mathrm{~h}$ had passed since ingestion, which was one reason for not administering fomepizole. The effects of fomepizole on haemodialysis withdrawal rate and shortening of haemodialysis period have not been completely clarified in patients with advanced acute kidney injury associated with ethylene glycol poisoning. Therefore, it is difficult to determine whether fomepizole would have been effective in our case. We consider that conventional treatments such as haemodialysis may be more useful than fomepizole in terms of cost benefits in patients with addiction for whom a long time had passed from ingestion or who have advanced renal injury. Despite this, there is no strong reason to deny the administration of fomepizole. Further, considering a previous report that a single dose of fomepizole may suffice if haemodialysis is initiated swiftly (Sidlak et al. 2021), efforts to obtain fomepizole at least after the first haemodialysis cycle could have been considered in our case.

\section{Conclusion}

We report a case of ethylene glycol poisoning that was treated using with a combination of ethanol and haemodialysis therapy instead of fomepizole. The effects of fomepizole on acute kidney injury due to ethylene glycol poisoning have not been determined. As the time period between ethylene glycol ingestion and treatment was at least $40 \mathrm{~h}$ in our case, the introduction of haemodialysis was reasonable and valid. Conventional treatments such as haemodialysis may be more useful than fomepizole in terms of cost benefits in patients with addiction for whom a long time had passed from ingestion or who have advanced renal injury. However, it is necessary to establish a system that enables rapid administration of specific antagonists such as fomepizole when required. It is also important to create an environment in which fomepizole can be administered promptly and carefully consider the cost benefits and indications for each case.

\section{Acknowledgement}

We would like to thank Editage (www.editage.com) for English language editing. 


\section{References}

[1] Amano K, Morita M, Ebihara T, Kato F, Kawada M, Usui A \& Nakata Y (2018) Fomepizole for ethylene glycol poisoning: two case reviews. Nihon Kyukyu Igakukai Zasshi 29, 12-17 (in Japanese).

[2] Basnayake BMDB, Wazil AWM, Nanayakkara N, Mahanama RMBSS, Premathilake PNS \& Galkaduwa KKMCDK (2019) Ethylene glycol intoxication following brake fluid ingestion complicated with unilateral facial nerve palsy: a case report. Journal of Medical Case Reports 13, 1-4. https://doi.org/10.1186/s13256-019-2139-z.

[3] Hovda KE \& Jacobsen D (2008) Expert opinion: fomepizole may ameliorate the need for hemodialysis in methanol poisoning. Human and Experimental Toxicology 27, 539-546. https://doi.org/10.1177/0960327108095992.

[4] Howard PH, Boethling RS, Jarvis WF, Meylan WM \& Michalenko EM (1991) Handbook of environmental degradation rates. 1st edn. Lewis Publishers, Chelsea, pp. 392-393. https://doi.org/10.1201/9780203719329-3.

[5] Kiyota K (2016) Antidote updates. Nippon Rinsho 74, 236-240 (in Japanese, abstract in English).

[6] Ono Y, Hata N, Sonoda Y \& Tohma Y (2017) Fomepizole alone therapy for a case of methanol intoxication. Nihon Kyukyu Igakukai Zasshi 28, 275-280 (in Japanese).

[7] Mégarbane B (2010) Treatment of patients with ethylene glycol or methanol poisoning: focus on fomepizole. Open Access Emergency Medicine 24, 67-75. https://doi.org/10.2147/OAEM.S5346.

[8] Nosaka H \& Satou K (2019) A case of severe ethylene glycol poisoning that was successfully treated with early administration of fomepizole and continuous renal replacement therapy. Nihon Toseki Igakkai Zasshi 52, 291-293 (in Japanese, abstract in English). https://doi.org/10.4009/jsdt.52.291.

[9] Rietjens SJ, de Lange DW \& Meulenbelt J (2014) Ethylene glycol or methanol intoxication: which antidote should be used, fomepizole or ethanol? Netherlands Journal of Medicine 72, 73-79.

[10] Roberts DM, Yates C, Megarbane B, Winchester JF, Maclaren R, Gosselin S, Nolin TD, Lavergne V, Hoffman RS, Ghannoum M \& EXTRIP Work Group (2015) Recommendations for the role of extracorporeal treatments in the management of acute methanol poisoning: a systematic review and consensus statement. Critical Care Medicine 43, 461-472. https://doi.org/10.1097/CCM.0000000000000708.

[11] Sidlak AM, Marino RT, Van Meerbeke JP \& Pizon AF (2021) Single versus continued dosing of fomepizole during hemodialysis in ethylene glycol toxicity. Clinical Toxicology 59, 106-110. https://doi.org/10.1080/15563650.2020.1770780.

[12] Tanasescu A, Macovel RA \& Tudosie MS (2014) Outcome of patients in acute poisoning with ethylene glycol-factors which may have influence on evolution. Journal of Medicine and Life 7, 81-86.

[13] Uchida H, Hashimoto T, Owada M, Sugawara T, Kawamura M \& Miyate Y (2011) Usefulness of osmolar gap in early diagnosis of case of ethylene glycol poisoning. Medical Journal of Iwate Prefectural Hospital 51, 29-32.

[14] Yamada T \& Nishio Y (2004) A case of ethylene glycol poisoning with severe metabolic acidosis and acute renal failure; successful treatment with continuous hemodiafiltration, hemodialysis and ethanol. Nihon Toseki Igakkai Zasshi 37, 169-173. https://doi.org/10.4009/jsdt.37.169. 